\title{
La situación de Mauretania a finales del Segundo Triunvirato e inicios del principado de Augusto
}

\author{
Luis AMELA VALVERDE ${ }^{1}$ \\ Grupo CEIPAC. Universidad de Barcelona \\ amelavalverde@gmail.com
}

\section{RESUMEN}

La situación de Mauretania tras la muerte de Boco II es uno de los temas más interesantes de la época final de la República en el norte de África. En el presente trabajo intentamos efectuar un acercamiento a esta interesante cuestión.

Palabras clave: Mauretania. Bogud. Boco II. Octaviano. Provincia.

\section{The situation of Mauretania in late II Triumvirate and early Augustan}

\begin{abstract}
The situation in Mauretania after the death of Bocchus II is one of the most interesting themes of the final period of the republic in northern Africa. In this paper we attempt to make an approach to this interesting question.
\end{abstract}

Key words: Mauretania. Bogud. Bocchus II. Octavian. province.

1 Este trabajo se ha realizado en el marco del Proyecto I+D+I 2009-2011 HAR 2011-24593. Agradezco al Prof. Dr. Ll. Pons Pujol la lectura y correcciones al presente texto. 
Mauretania, más en concreto, los dos reinos mauretanos, ${ }^{2}$ gobernados al menos desde el año 49 a.C. por Bogud en la parte occidental y por Boco II en la parte oriental, ${ }^{3}$ ambos seguramente relacionados con Boco I (118?-80? a.C.), aunque se desconoce la conexión exacta, ${ }^{4}$ el monarca que participó en la guerra de Yugurta (111-105 a.C.). En este trabajo queremos tratar los años finales de la dinastía indígena de Mauretania, involucrada en los conflictos internos que azotaron los últimos años de la República Romana, antes de la subida al trono norteafricano de Juba II (25 a.C.-23 d.C.) por obra del emperador Augusto (27 a.C.- 14 d.C.).

Boco II tendría como capital $\mathrm{Iol},{ }^{5}$ mientras que Bogud tendría su sede en la ciudad de Volubilis. ${ }^{6}$ Ambos reinos estaban separados por el río Mulucha (Moulouya) ${ }^{7}$ este río era antiguamente la frontera entre Mauretania y Numidia, ${ }^{8}$ por lo que puede observarse la expansión del reino mauretano hacia Oriente a expensas de sus vecinos, ya iniciada tras la finalización de la guerra de Yugurta. ${ }^{9}$

Al inicio de la guerra civil (49 a.C.) entre C. Julio César (cos. I 59 a.C.) y el Senado, tanto Bogud como Boco II se declararon por el primero, ${ }^{10}$ con lo que rompieron sus lazos con la familia de L. Cornelio Sila (cos. I 88 a.C.), puesto que los reyes númidas, sus rivales, debido a sus relaciones con la familia de los Escipiones, militaron en el bando pompeyano. ${ }^{11}$

Después de la muerte de César (44 a.C.), Bogud se puso al servicio de Marco Antonio (cos. I 44 a.C.), como anteriormente había estado al servicio del Dictador, mientras que Boco II se decantó por C. Julio Octaviano (cos. I 43 a.C.), ${ }^{12}$ sobrino e hijo adoptivo de César. El hecho de que Boco II se uniese a Octaviano se debía menos por el control efímero de este último sobre África en el año 43 a.C. que por la fidelidad del rey mauretano a la memoria de César. ${ }^{13}$ Por su parte, Gozalbes considera que Bogud se pasó al bando antoniniano debido a que Boco II apoyaba a Octaviano. ${ }^{14}$

Por una carta de la correspondencia de M. Tulio Cicerón (cos. 63 a.C.), conocemos que Marco Antonio tenía en su ejército en mayo del año 43 a.C. jinetes mauros, ${ }^{15}$

\footnotetext{
2 Como indica PAVIS D’Escurac 1982, 223, la división entre ambas áreas debió de ser tan profunda que, cuando el territorio fue definitivamente anexionado por Roma, se dividió en dos provincias: Mauretania Cesariana y Mauretania Tingitana.

3 Plin. NH 5, 19. Str. 17, 3, 7.

4 Roller 2003, 55.

5 Mela, 1, 29. Solin. 25, 16.

6 Roller 2003, 56.

7 Plin. NH 5, 2, 19, quien dice de Mauretania que: diu regum nomina optinuere, ut Bogutiana appellaretur extuma, itemque Bocchi quae nunc Caesariensis (Gsell 1928a, 273 n. 4).

8 Sall. Iug. 92, 5.

9 Sall. Bell. Iug. 110, 8.

10 Dio Cass. 42, 42, 7.

11 Coltelloni-Tranoy 1997a, 65.

12 Gsell 1928b, 199; Romanelli 1959, 149; Gascou 1974, 67; Ghazi-Ben Maïssa 1996, 1416; Gozalbes 1997, 30; Coltelloni-Trannoy 1999, 71.

13 Coltelloni-Tranoy 1997a, 64.

14 Gozalbes 1997, 30.

15 Cic. Fam. 10, 30, 3.
} 
que Bogud le habría enviado. ${ }^{16}$ Esto es interpretado por Coltelloni-Trannoy ${ }^{17}$ como muestra de la precocidad de Bogud en aliarse con Marco Antonio, aunque Romanelli señala igualmente la posibilidad de que los hubiera heredado del ejército de César. ${ }^{18}$

En el mes de junio del mismo año, Asinio Polión, gobernador de la Hispania Ulterior, señala a Cicerón que su cuestor, L. Cornelio Balbo (cos. suff. 32 a.C.?), pasó a los estados de Bogud con la caja provincial (sin olvidarse el producto de sus rapiñas). ${ }^{19}$ No se trataba de que el gobernador se opusiera a la propaganda antoniniana de su cuestor, ${ }^{20}$ sino que Polión consideraba que no había llegado el momento oportuno de enseñar sus cartas. ${ }^{21}$

Como indica Coltelloni-Trannoy ${ }^{22}$, no se puede ignorar las decisiones en serie que, en el espacio de tres meses, valen a Marco Antonio el apoyo de un rey, de un gobernador y de un miembro de una de las más prestigiosas familias de Gades, los tres ligados, en diversos grados, a los confines occidentales del Imperio. Para comprender esta conjunción de intereses, hay que recordar las relaciones ancestrales que unían las dos riberas del Estrecho de Gibraltar, fuesen pacíficas y comerciales, o a veces de carácter belicoso. Para esta investigadora, los romanos de Hispania intentaron ensayar de conciliarse a los reyes turbulentos de estos países africanos incluyéndolos en sus redes de clientela, como muestra la presencia de los posteriores reyes Juba II (25 a.C. -23 d.C) y Ptolomeo (23 d.C.- 40 d.C.) en Carthago Nova ${ }^{23}$ y Gades. ${ }^{24}$

Asimismo, el vínculo entre Boco II y Octaviano se debería a que, si bien había heredado parte del antiguo reino de Boco I, englobaba también comunidades númidas, y que Boco II actuaría en interés de sus nuevos súbditos. ${ }^{25} \mathrm{El}$ conflicto entre Octaviano y Marco Antonio ofrecía un medio de expresión ideal a la rivalidad que en adelante separaría a ambos monarcas mauretanos. ${ }^{26}$ Ahora bien, la causa original de la hostilidad entre ambos personajes nos es desconocida; quizás, el hecho de que la gran parte del antiguo reino de Numidia había sido anexionado por Roma fuese un detonante, puesto que, para evitar este final, se intentaría reunir todo el territorio de Mauretania bajo un solo cetro.

Por tanto, la rivalidad en el mundo romano llegó asímismo al seno de la familia real mauretana. En tiempos de la Guerra de Perusa (41 a.C.), C. Carrinas (cos. 43 a.C.) era el gobernador de la Hispania Ulterior a nombre de Octaviano; por Apiano sabemos que. L. Antonio (cos. 41 a.C.), el hermano de Marco Antonio, ordenó a Boco que lo atacase, ${ }^{27}$ hecho que Schulten niega, ${ }^{28}$ aunque pudo existir la sugerencia. De

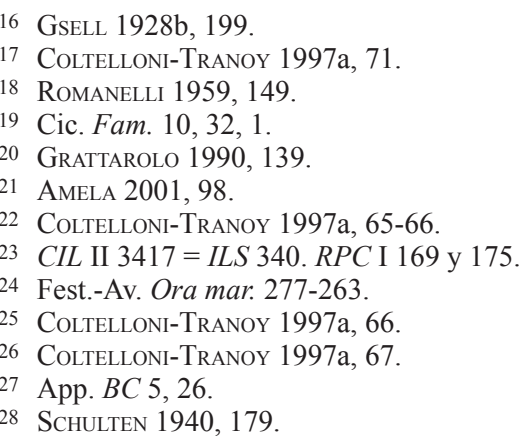


hecho, existe un error en el nombre del monarca mauretano, ya que es Bogud quien intervendría, debido a que desde su reino era más fácil pasar a la Península y demostrar su compromiso con Marco Antonio. ${ }^{29}$ Desgraciadamente, lo breve de la noticia no nos permite obtener conclusiones claras.

Que las relaciones entre las dos costas del Estrecho de Gibraltar por este tiempo no fueron precisamente pacíficas lo muestra la circunstancia de que Bogud, rey de Mauretania, en el año 38 a.C., fuese por incitación de Marco Antonio ${ }^{30}$ o por iniciativa propia, ${ }^{31}$ invadió Hispania, donde causó mucho daño a la vez que también los sufrió. ${ }^{32}$ Este ataque sería llevado a cabo posiblemente aprovechando Bogud la circunstancia de que el gobernador de la provincia, Cn. Domicio Calvino (cos. II 40 a.C.) se encontraba ocupado en el norte, luchando contra los cerretanos. ${ }^{33}$

Coltelloni-Trannoy considera que el ataque de Bogud muestra que la fides exigida por el sistema de clientela era mal comprendida por los monarcas norteafricanos, al considerar esta investigadora que los reyes mauritanos estarían ligados por esta institución a Octaviano, como heredero de César. ${ }^{34}$ En realidad, el cambio de bando que se registra durante este tiempo obedece únicamente a los intereses individuales de cada uno de los monarcas, sin que exista mecanismo clientilicio alguno, sino que según se desarrollaban los acontecimientos así se actuaba.

Gsell considera que Bogud atacó por instigación de Sexto Pompeyo, el hijo menor de Cn. Pompeyo Magno (cos. I 70 a.C.), quien en este momento dominaba los mares desde su base en Sicilia, puesto que Marco Antonio no quería tener problemas con Octaviano. ${ }^{35}$ Romanelli señala, de manera acertada, que Gsell hierra, puesto que los monarcas mauretanos estaban normalmente en contra de Pompeyo Magno y de los pompeyanos. ${ }^{36}$

Las semejanzas en el ataque contra Hispania en el año 41 a.C., transmitido por Apiano, y el del año 38 a.C., por Dión Casio, ha ocasionado que varios estudiosos considerasen que se trata en realidad de la misma agresión. De este modo, Blázquez piensa que Marco Antonio incitó a Bogud a atacar a Carrinas, pero que no lo llevaría a la práctica hasta el año 38 a.C. ${ }^{37}$ Por su parte, Freyburger y Roddaz consideran que Dión Casio hierra en la cronología, y remontan este hecho al año 41 a.C., como ya hemos indicado y, evidentemente, sería L. Antonio y no Marco Antonio el incitador de Bogud. ${ }^{38}$

El problema que plantean las fuentes que nos han llegado es que son siempre citas esquemáticas, sin desarrollar, lo que induce a ciertos investigadores a considerar que la información contenida puede ser errónea. Quizás aconteciera que Bogud recibiera

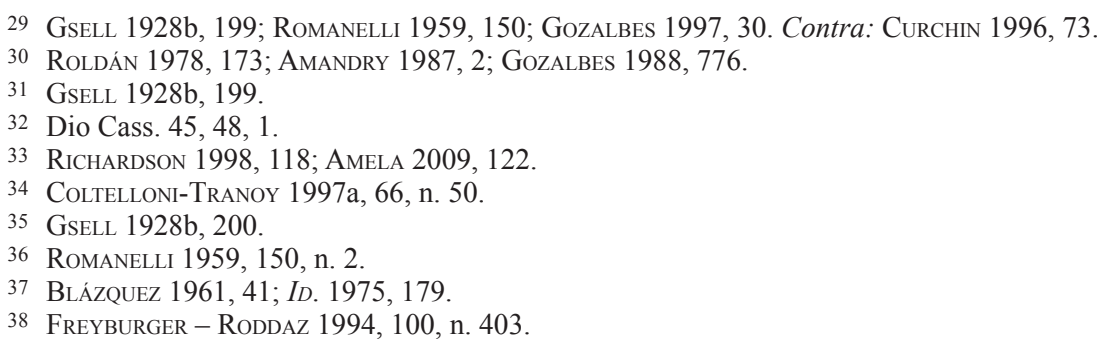


la orden de atacar Hispania en el año 41 a.C. pero que, por diversos motivos, no lo hizo hasta el año 38 a.C.; o se trata de dos acciones diferentes. Difícilmente lo sabremos.

Una aislada noticia de Porfirio señala que Bogud asedió durante largo tiempo el muy rico templo de Hércules, en Gades,${ }^{39}$ que pudiera relacionarse con este evento: ${ }^{40}$ Porfirio señala que el Herakleion poseía grandes riquezas, lo que indica porqué procedía el monarca mauritano. El asedio se prolongó durante mucho tiempo, por cuya causa escasearon las víctimas para el sacrificio del templo, y se alude a un supuesto prodigio entonces acaecido. El sacerdote del templo de Hércules, no sabiendo qué hacer, tuvo el sueño siguiente: le pareció que se encontraba en medio de las columnas de Hércules, y que enfrente del altar estaba posado un pájaro que intentaba volar hacia él; levantó el vuelo y vino a sus manos; y con la sangre del pájaro roció el altar (una costumbre que era diaria). Después de esta visión, se levantó al amanecer, se dirigió al altar, y como en el sueño, dirigió la vista a la torre; vio un pájaro como el del sueño, y confiando en que se realizaría la visión, se puso en pie. Bajando el ave, se posó sobre el altar, se entregó a manos del primer sacerdote y así fue sacrificada y se roció el altar.

Quizás el objetivo principal de Bogud fue el templo de Hércules. ${ }^{41}$ Éste ya había sido saqueado por el general cartaginés Magón en el año 206 a.C., ${ }^{42}$ y por M. Terencio Varrón (vir pr.) en el año 49 a.C., aunque en esta ocasión César devolvió el tesoro, ${ }^{43}$ y luego por el propio César, ${ }^{44}$ aunque Blázquez considera que esto último no es probable, ${ }^{45}$ pero ésta es una suposición gratuita. Una actuación que no le separaba en absoluto de un acto de piratería. ${ }^{46} \mathrm{El}$ asedio no lo padeció exactamente el $\mathrm{He}$ rakleion, sino la ciudad insular de Gades, donde el templo estaba situado en su zona oriental. ${ }^{47}$ Este fue un error gravísimo de Bogud, pues se enajenó la enemistad con la aristocracia comercial gaditana, que tenía mucha influencia en la vecina África. ${ }^{48}$

Sea como fuere, puede afirmarse que en el año 38 a.C. Bogud atacó Hispania, pero se encontró con una eventualidad inesperada. Los habitantes de Tingi (Tánger, Marruecos), la principal ciudad del reino de Bogud, ${ }^{49}$ se sublevaron, por lo que Bogud se vio obligado a volver a África.

Bogud no pudo restablecer la situación, debido a que los partidarios de Octaviano en Hispania, junto con Boco, el rey de la Mauretania Oriental, que se unió a los primeros, le derrotaron, ${ }^{50}$ en una evidente operación de tenaza. ${ }^{51}$ Muy probablemente,

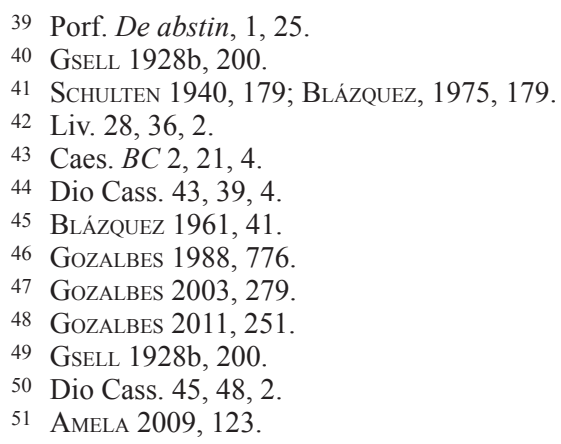


Boco no sería ajeno a la rebelión de Tingi ${ }^{52}$ y el ataque contra Gades, el mayor centro comercial de la región, desde luego no favoreció las aspiraciones de Bogud. ${ }^{53}$ Asimismo, seguramente participaron tropas de Octaviano, ${ }^{54}$ como parece lógico, además de deducirse del testimonio de Dión Casio; de esta manera sería erróneo el testimonio de Plinio de que la primera vez que aparecieron tropas romanas en Mauretania fue bajo el gobierno del emperador Claudio (41-54 d.C.), ${ }^{55}$ en relación con la revuelta de Edemón. ${ }^{56}$

Bogud no tuvo más remedio que huir junto a Marco Antonio en Oriente, apoderándose Boco de su reino, lo que le fue confirmado por Octaviano, ${ }^{57}$ con lo que su estado se extendía entonces desde el Atlántico hasta el río Ampsaga (Oued El Kébir). ${ }^{58}$ Evidentemente, la ampliación de los dominios de Boco estaba relacionada con su apoyo a Octaviano, el dueño de Occidente. Esta rebelión contra el poder real indica la escasa popularidad de Bogud y los deseos de libertad de las ciudades más prósperas del NO de la Mauretania. ${ }^{59}$ Pero, como señala Madjoub, ${ }^{60}$ la anexión del territorio de Bogud por Boco II no dejó huella en las fuentes, ${ }^{61}$ por lo que quizás tuvo dificultades en controlar el nuevo territorio.

En este sentido, en Tingi y su zona de influencia no se ha detectado ningún rastro de devastación. Por el contrario, en Tamuda, Sidi Abdselam el Bhar, Kouass, Zilil, Siga y Lixus se documentan niveles de destrucción, pero que no se pueden atribuir exactamente a un momento histórico concreto. ${ }^{62}$

Ciertamente, es muy difícil poder atribuir algún rastro de destrucción para este momento histórico concreto. De esta forma, actualmente, en Tamuda se ha detectado únicamente un abandono no planificado de la ciudad, pero $c a$. el año 100 a.C. ${ }^{63}$ Por su parte, Zilil es un buen ejemplo de los problemas que se pueden dar a nivel arqueológico, pues si bien en un principio se creía que esta población había sido destruida a finales del s. II a.C., a partir de los materiales encontrados, actualmente se ha retrotraído la fecha al s. III a.C. ${ }^{64}$ Por tanto, es bastante difícil poder establecer conclusiones satisfactorias.

52 Romanelli 1959, 150; Decret - Fantar 1998, 163.

53 Gozalbes 2011, 251.

54 Romanelli 1959, 150.

55 Plin. NH 5, 19.

56 Madjoub 2000, 1726.

57 Dio Cass. 45, 48, 3.

58 Gsell 1928b, 200; Mazard 1953, 13; ID. 1955, 57 y 60; Romanelli 1959, 150 y 161; Benabou 1976, 48; Mackie, 1983, 333; Decret - Fantar 1998, 163.

59 Gozalbes 1997, 30.

60 Madjoub 2000, 1726.

61 Cf. Plin. $N H$ 5, 16. Str. 17, 3, 7.

62 Madjoub 2000, 1730; Callegarin 2002, 29-30; Gozalbes 2003, 280.

63 Bernal et alii 2011, 302 y 308.

64 Kbiri-Alaoul 2004, 205. 
A los tingitanos, en recompensa por su oportuna rebelión, se les concedió el derecho de ciudad ${ }^{65}$ fuese la ciudadanía romana ${ }^{66}$ o latina, ${ }^{67}$ es decir, su conversión a municipio; ${ }^{68} \mathrm{o}$ incluso erróneamente (por la mala lectura de una moneda, vid infra) su conversión a una colonia romana, ${ }^{69}$ aunque Dión Casio nada dice de la concesión de este último estatuto jurídico. ${ }^{70}$ Octaviano excluyó pues a Tingi del beneficio obtenido por Boco, ya que sería impensable que tuviera autoridad sobre una población de ciudadanos romanos. ${ }^{71}$ Por tanto, esta ciudad se incorporaría a la Hispania Ulterior. ${ }^{72}$

Bogud, tras su huída del continente africano, sirvió fielmente a Marco Antonio, y es conocida su participación en la guerra final contra Octaviano. ${ }^{73}$ En la primavera del año 31 a.C., antes de la batalla de Actium, Bogud fue capturado y condenado a muerte por Agripa en Methone, localidad de Mesenia ${ }^{74}$ ciudad de la que era el comandante.

A su muerte en el año 33 a.C., Boco II legó todo el territorio, tanto la Mauretania occidental como la oriental, al pueblo de Roma, ${ }^{75}$ probablemente, como indica Gsell, por no tener herederos, aunque este mismo autor señala que Boco II tenía hijos en el año 45 a.C. ${ }^{76}$ si es que este dato no es inexacto, ${ }^{77}$ ya que como indica ColtelloniTrannoy, ${ }^{78}$ el que éstos fueran enviados a Sexto Pompeyo parece apuntar más al príncipe númida Arabión.

Entre la muerte de Boco en 33 a.C. y la instalación de Juba II en el trono de Mauretania en el año 25 a.C., nos encontramos un período obscuro en la historia de este territorio, tradicionalmente llamado el "interregno", un término que Pavis d'Escurac no considera acertado. ${ }^{79} \mathrm{El}$ problema principal es considerar si Mauretania se convirtió o no en provincia romana, lo que ha dividido a la investigación actual.

El que Boco II dejara su reino en herencia a Roma y que ésta la aceptó puede apoyarse en dos hechos claros: la fabricación de monedas mauretanas a nombre de Octaviano ${ }^{80}$ y la colonización. La circulación de estas monedas muestra que el territorio estaría en manos romanas y que "el reino protegido" emitía sus propias piezas. ${ }^{81}$ Ciertamente, en estas mismas monedas no figura ningún nombre de magistrado, sino

65 Dio Cass. $45,48,3$.

66 Gsell 1928b, 200; Romanelli 1959, 150; Gascou 1974, 67 y 70; Pavis d’Escurac 1982, 223; Mackie 1983, 343; Amandry 1987, 2; Hamdoune 1994, 83; Gozalbes 1997, 32 ; Decret - Fantar 1998, 163; Rhorfi 2002, 2147-2148 у 2159; Amela 2009, 124.

67 EuzenNat 1996, 337.

68 Gascou 1974, 6 y 708; Amandry 1987, 2; Burnett et alii 1992, 210 ; Coltelloni-Trannoy $2005,132$.

69 CArcopino 1943, 176; BlázQuez 1961, 42; Rhorfi 1998, 304 y 309; ID. 2002, 2159-2161.

70 Gascou 1974, 67.

71 Gascou 1974, 67.

72 Blázquez 1961, 42; Hamdoune 1994, 84; Gozalbes 2003, 281.

73 Plut. Ant. 61, 2, donde el nombre de Boco ha de ser reemplazado por el de Bogud. GseLL 1928b, 200; Romanelli 1959, 150 n. 4; Freyburger - Roddaz 1994, 100, n. 403.

74 Dio 50. 11, 3. Porf. De abstin., 1, 25. Str. 8, 4, 3.

75 Dio Cass. 49, 43, 7. Benabou 1976, 48; Gozalbes 1997, 319.

76 Dio Cass. 43, 36, 1 .

77 GSELl 1928b, 200.

78 Coltelloni-Trannoy 1997a, 79, n. 116; ID. 1997b, 21, n. 11.

79 Pavis D'Escurac 1982, 222.

80 RPC I 877-879.

81 Pavis D'Escurac 1982, 222. 
únicamente el de Octaviano, lo que indica que Mauretania, como entidad política autónoma, no existiría. ${ }^{82}$ En cualquier caso, parece que hay que descartar la existencia de un vacío de poder en la región.

Octaviano fundó doce colonias, como lo probarían los epítetos que figuran en las inscripciones, pobladas por veteranos, como atestiguan sus nombres, ${ }^{83}$ en su mayoría en la costa o a poca distancia de ésta: tres en el Oeste, llevando el título de Iulia, y nueve en el Este, llevando, por lo que nos es conocido, el título de Iulia Augusta. ${ }^{84}$ Así, en el Oeste, encontramos las siguientes colonias: Iulia Constantia Zilil (Dchar Jdid, cercano a Arcila), ${ }^{85}$ Iulia Campestris Babba (de ubicación desconocida), ${ }^{86}$ e Iulia Valentia Banasa (Sidi Ali bou Djenoun). ${ }^{87}$ Por otro lado, en el Este, en la costa, se hallan: Cartennae (Ténès), ${ }^{88}$ Gunugu (Koubba de Sidi Brahim, cerca de Gouraya) ${ }^{89}$ Rusazus (Azeffoun),${ }^{90}$ Rusguniae (Cap Matifou) ${ }^{91}{ }^{91}$ Saldae (Bougie),${ }^{92}$ Igilgili (Djidjeli); ${ }^{93}$ en el interior: Zucchabar (Miliana), ${ }^{94}$ Aquae Calidae (Hammamm Rhiga), ${ }^{95}$ y Tubusuptu (Tiklat). ${ }^{96} \mathrm{La}$ diferencia de título parece indicar que estas colonias fueron fundadas en dos grupos: las colonias del Oeste antes del 27 a.C., las del Este, entre 27 y 25 a.C. ${ }^{97}$ aunque también se mantiene que fueron creadas por el emperador Claudio (41-54 d.C.) ${ }^{98}$ Como dicen Decret y Fantar, ${ }^{99}$ las colonias responderían a los habituales factores comerciales, económicos e incluso estratégicos, en las que realmente no serían más que auténticas "cabezas de puente". 100

82 Coltelloni-Trannoy 1997b, 25.

83 Romanelli 1959, 162 y 201; Benabou 1976, 55; Pavis d’Escurac 1982, 224; García-Gelabert 1994, 1203; Coltelloni-Trannoy 1997b, 24; ID. 2005, 135; Decret - Fantar 1998, 163.

${ }^{84}$ Gsell 1928b, 201-205; Benabou 1976, 55; Mackie 1983, 337; Amandry 1987, 2; ID. 2000: 54; Coltelloni-Trannoy 1997b, 124; ID. 2005, 135; DeCret - Fantar 1998, 163; Corbier - Griesheimer 2005, 50; Roller 2003, 96; Briand-Ponsart - Hugoniat 2006, 49; Gozalbes 2011, 253.

85 Plin. $N H 5,1,2$.

86 Plin. $N H 5,5$.

$87 A E$ 1941, 79. Gsell 1928b, 202-203; Mazard 1953, 19; Romanelli 1959, 201-202; Grant 1969, 223; Mackie 1983, 337; Amandry 1984, 93; ID. 1987, 2; ID. 1991-1993, 239; ID. 2000, 54; GarCía-Gelabert 1994, 1203; Coltelloni-Trannoy 1997b, 124; ID. 2005, 135; Decret - Fantar 1998, 164; Hugoniot 2000, 51; Corbier - GriesheImer 2005, 51; Le BoHec 2005, 51.

88 Plin. NH 5, 20.

89 Plin. $N H 5,1,20$.

90 Plin. NH 5, 20. $A E$ 1921, 16.

91 Plin. $N H$ 5, 20. $A E$ 1956, 160.

92 Plin. NH 5, 1, 20. CIL VIII 8829. CIL VIII $20683=I L S$ 649. CIL VIII $8931=I L S 662 . C I L$ VIII 8933.

93 Plin. $N H$ 5, 20.

94 Plin. NH 5, 1, 20. AE 1940, 20.

95 Plin. $N H 5,1,21$.

96 Plin. NH 5, 21. CIL VIII $8837=$ ILS 6303; AE 1934 39; AE 1967 641. Gsell 1928b, 202; MAZARD 1953, 19; Romanelli 1959, 202-204; Benabou 1976, 55-56; Pavis d'Escurac 1982, 222-223; Mackie 1983, 338; Amandry 1987, 2; ID.1991-1993, 239; ID. 2000, 54; García-Gelabert 1994, 1203; Coltelloni-Trannoy 1997b, 124; ID. 2005, 135; Decret - Fantar 1998, 164; Hugoniot 2000, 51; Roller 2003, 96; Corbier GriesheIMER 2005, 50-51; Le BoHeC 2005, 51.

97 Gsell 1928b, 202; Romanelli 1959, 201-202; Grant 1969, 60; Benabou 1976, 55; Mackie 1983, 338 y 340; Amandry 1987, 2; ID. 1991-1993, 239; ID. 2000, 54; Coltelloni-Trannoy 1997b, 124; Laporte 2002, 562-563; GozalBes 2011, 253.

98 ROLLER 2003, 96.

99 DeCRET - FANTAR 1998, 164.

100 Gsell 1928b, 204; Pavis d’Escurac 1982, 224; Briand-Ponsart - Hugoniat 2006, 49. 
Como señala Laporte, ${ }^{101}$ la fundación de las tres colonias en la futura Mauretania Tingitana responde probablemente a la rivalidad entre Octaviano y Marco Antonio. En el año 36 a.C. Marco Antonio sufre una derrota en Partia; en el año 33 a.C., Boco II fallece y Octaviano anexiona las dos Mauretanias. La comparación entre ambos personajes romanos, en que el último aumenta el territorio romano mientras que el primero, mediante las denominadas "Donaciones de Alejandría", efectúa justamente lo contrario, pretendía acentuar el prestigio de Octaviano sobre Marco Antonio. Sería en esta coyuntura en que estas colonias serían fundadas desde Hispania.

Por el contrario, las colonias fundadas en la futura Mauretania Caesariense obedecen a la gran ola de desmovilización que siguió a la campaña de Actium. ${ }^{102}$ Ciertamente, puede comprenderse que estas nueve colonias se fundaron en un espacio temporal de dos años.

Tingi no está citada entre las colonias fundadas. Pero en una serie de monedas, datadas en un principio en época preaugustea, figuraría el título de Colonia Iulia Tingi. ${ }^{103}$ Así, era lógico pensar, como ya hemos indicado, que Octaviano, al crear las tres colonias del oeste de la Mauretania, elevaría a Tingi al mismo estatuto. ${ }^{104}$ De los textos de Plinio y de Estrabón, ${ }^{105}$ parecería desprenderse que se instalaron veteranos en Tingi y que, paralelamente, los ciudadanos de Tingi fueron instalados en Hispania en la colonia de Iulia Traducta con los habitantes venidos de Zilil, evacuada para hacer lugar a los colonos romanos. ${ }^{106}$ De esta forma, Tingi se convertiría en colonia romana entre los años 33 y 25 a.C. ${ }^{107}$ Pero toda la reconstrucción anterior ha quedado desacreditada debido a que una nueva lectura de las monedas ha eliminado el título de colonia. ${ }^{108}$

Tingi así como las otras doce colonias fueron transferidas administrativamente a la provincia de la Bética. ${ }^{109}$ En cuanto a la fecha de la creación de la provincia de la Bética, y su relación con las colonias augusteas en Mauretania, si la primera se creó posteriormente a la entrega de Mauretania a Juba II, estas colonias dependerían entonces de la provincia de Hispania Ulterior. Después de la deposición de Ptolomeo por Calígula y la anexión del reino de Mauretania, Claudio creó las provincias de Mauretania Tingitana y Mauretania Cesariana con Tingi e Iol/Caesarea por capitales. Parece ser que en este momento se efectuó una deductio en Tingi, debido a la aparición del título de Colonia Claudia Tingi que nos es conocido epigráficamente. ${ }^{110}$

Como indica Laporte, ${ }^{111}$ parecería paradójico que se estableciera una serie de colonias en una provincia que poco después iba a ser entregada a un monarca extranjero.

101 LAPORTE 2002, 562.

102 LAPORTE 2002, 563.

103 RPC I 860.

104 Blázquez 1961, 42; Gascou 1974, 68 y 70; Pavis d’Escurac 1982, 223; Amandry 1984, 92; ID. 1987, 2; Coltelloni-Trannoy 1997b, 124; Hugoniot 2000, 51.

105 Plin. NH 5, 2; Str. 3, 8.

106 Amandry 1987, 2; Coltelloni-Tranoy 1997a, 66, n. 49.

107 BuRnetT et alii 1992, 210.

108 BuRNETT et alii 1998, 16.

109 Plin. NH 5, 5.

110 CIL VI 31870

111 Laporte 2002, 563. 
Este investigador no sabe decidirse si ello correspondería a una política de romanización brutalmente interrumpida o a una toma de precaución ante la reconstitución de un reino teóricamente distinto del territorio romano.

Octaviano decidió una solución intermedia. ${ }^{112}$ En el año 25 a.C., Mauretania reencontró su independencia y se convirtió en el reino de Juba II, el hijo del homónimo monarca númida vencido en Thapsus (46 a.C.), un rey "indígena" fiel y sumiso a Roma, que cumpliera con lo que se esperaba de él: controlar el territorio. ${ }^{113}$

Parece claro, como indica el testimonio de Dión Casio, ${ }^{114}$ que Mauretania estaba en dependencia directa de Octaviano y de Roma, lo que ha sido interpretado como la prueba de un lazo personal entre Octaviano y los jefes de confederaciones tribales mauros. Asímismo, cuando Augusto procede a la entronización de Juba II, Tácito señala que Juba II recibió los mauros como regalo del pueblo romano. ${ }^{115}$ Así pues, Roma es la que dirige los destinos de Mauretania.

Así, Coltelloni-Trannoy, a partir de que Dión Casio señala que "Octavio se hizo dar en persona el reino, pero lo inscribió en el número de provincias", ${ }^{116}$ muestra el papel determinante de Octaviano en la decisión de la anexión del territorio. De hecho, es en el año 33 a.C., justo en el momento en que muere Boco, cuando se produce la ruptura entre Octaviano y Marco Antonio, con lo que el primero podía hacer coincidir el interés de la res publica (y el suyo propio) con la situación de hecho instaurada en el año 38 a.C. Es decir, desde el día que Masinisa (206-148 a.C.) hubo confiado a P. Cornelio Escipión el Africano (cos. I 205 a.C.) el cuidado de arreglar su sucesión, ${ }^{117}$ Roma se consideraba legalmente responsable del reino númida, parte del cual ahora estaba en manos mauretanas.

De hecho, el período posterior a la muerte de Boco II podría considerarse como una especie de anexión abortada. El reino originario de este monarca no cayó en manos de Roma debido a un conflicto bélico, sino que más bien éste fue embargado por Octaviano. ${ }^{118}$

Se ha planteado la existencia de un testamento efectuado por Boco II, ${ }^{119}$ lo cual no sería de extrañar, pues ciertos monarcas orientales habían dejado su reino al pueblo romano, sea por ausencia de heredero o por desbaratar los proyectos de ambiciosos indeseables; así ocurrió con los casos de los reinos de Pérgamo, la Cirenaica y Bitinia. Reencontramos parcialmente esta situación en Mauretania, donde el período que siguió a la muerte de Boco II es dominado por un vacío político evidente: ningún príncipe de la casa real o jefe de tribu parece reclamar la sucesión, fuese a la muerte de Boco II o al advenimiento de Juba II. Sea como fuere, hay una diferencia capital: es Octaviano quien se encarga de regular la sucesión, en lugar del Senado, órgano de

112 Benabou 1976, 49; Coltelloni-Trannoy 2005, 134.

113 Romanelli 1959, 162; Benabou 1976, 49.

114 Dio Cass. 50, 6, 4.

115 Tac. Ann. 4, 5. Pavis D’Escurac 1982, 222.

116 Dio Cass. 49, 43, 7; Coltelloni-Trannoy 1997a, 78.

117 App. Lib. 106.

118 Coltelloni-Tranoy 1997a, 78-79.

119 Gsell 1928b, 200; Romanelli 1959, 150; Coltelloni-Trannoy 1997b, 20; Hugoniot 2000, 49; BriandPonsart - Hugoniat 2006, 49. 
la política exterior, ya que es él quien toma la decisión de transferir el reino dentro del número de provincias. ${ }^{120}$

Coltelloni-Trannoy ${ }^{121}$ se pregunta si no estamos ante una etapa suplementaria de la revolución legislativa que, en las monarquías orientales, había marcado el derecho helenístico, al inventar la transmisión del poder por vía de la herencia, sobre el modelo de los principios del derecho privado. Boco II pudo inspirarse en este derecho para marcar su fidelidad a su patrón y le legó, en un último officium, el conjunto de tierras reales, y teniendo en cuenta que dentro de los cánones de las relaciones patrón-cliente romanas estaba dejar una parte de sus bienes a su muerte al primero.

Este gesto que Octaviano no está todavía en posición de aceptar se convertirá en usual, puesto que todos los sujetos del Imperio, beneficiarios virtuales o efectivos del princeps, legaron una parte de sus bienes a este último, en función de dos innovaciones mayores en el derecho sucesorio romano: la creación de la uicesima hereditatium, y la institución del heredero, realizada a favor del emperador. ${ }^{122}$

Coltelloni-Trannoy ${ }^{123}$ considera que el caso mauretano, en una época precoz donde se van ubicando los poderes y las situaciones que preparan la posición del futuro Augusto, es un precedente de ciertas medidas jurídicas que serán fijadas en el Imperio. La anexión del reino de Boco II al pueblo romano, por iniciativa de Octaviano, podría situarse en el punto de convergencia de dos lógicas: la del sistema de patronato que, ya con Masinisa, había conferido una autoridad considerable al patrón del rey en el caso de la sucesión, y que está siempre en concordancia con decisiones políticas mayores; y la del derecho romano tras una gestación de dos siglos, y que se encamina desde el final de la República hacia la adopción de las instituciones helenísticas, que transpone el cuadro antiguo y muy vivaz del sistema de clientelas, vigente hasta la fecha.

La decisión de Escipión el Africano concerniente a la sucesión de Masinisa, no es más que el eco del comportamiento de Octaviano en lo concerniente con la sucesión de Boco II: entre estos dos actos concerniente a patrones e importantes clientes, un siglo entero ha transcurrido en el cual se conjuga el peso de las clientelas romanas y el de la integración jurídica progresiva de los reinos africanos.

Boco II murió en el año 33 a.C., aparentemente sin herederos, creando un vacío de poder. Lo que pasó es incierto, y las dos únicas fuentes que tenemos son inútiles. Estrabón señala que después de las muertes de Bocco II y Bogud, Juba II heredó el reino. ${ }^{124}$ Este testimonio tiene poco valor: Bogud y Boco II murieron, como hemos visto, con años de diferencia. Y no parece que Juba II, que tenía en el año 33 a.C. quince años de edad, heredase el reino. ${ }^{125}$

Más explícita es la noticia de Dión Casio quien dice que cuando Boco II murió, Octaviano convirtió su territorio en una provincia romana. ${ }^{126}$ Esto ha originado una

120 Coltelloni-Trannoy 1997a, 79; ID. 1997b, 21.

121 Coltelloni-Trannoy 1997a, 79; ID. 1997b, 20-21.

122 Coltelloni-Trannoy 1997a, 79-80.

123 Coltelloni-Trannoy 1997a, 80; ID. 1997b, 21-22.

124 Str. 17, 3, 7.

125 Roller 2003, 95.

126 Dio Cass. 49, 43, 7. 
amplia polémica entre los historiadores. Lo más simple es que Dión Casio de alguna manera olvidó todo sobre la monarquía mauretana, habría confundido la muerte de Boco con la creación de la provincia de Mauretania setenta años más tarde. Los errores de cronología y combinación de acontecimientos diferentes son notorios en Dión Casio, pero posteriormente sitúa a Juba II en el reino correcto, así que su exposición sobre el estatuto de Mauretania en el año 33 a.C. puede ser considerado seriamente.

La existencia de una provincia de Mauretania ha sido considerada improbable por Roller, ${ }^{127}$ dado que el propio Augusto no la menciona como tal en su lista de aquéllas que le habían apoyado en Actium, ${ }^{128}$ y la versión de Dión Casio de la misma lista incluye entre los aliados de Octaviano "éstos pertenecientes a Bogud y Boco", ${ }^{129}$ que difícilmente podrían indicar la existencia de un estatuto provincial. Por otra parte, Mauretania está notoriamente ausente de la extensa lista de provincias en el asentamiento del año 27 a.C. ${ }^{130}$ No se conoce ningún magistrado romano o gobernador, y la entrega del territorio a Juba ocho años después significaría una reversión del estatuto de provincia a reino cliente que probablemente ofendería a más de uno.

Por tanto, sería más fácil considerar que Dión Casio cometió un error, al menos de su análisis de la situación contemporánea. Pero una moneda de Juba II con la doble fecha 48/5 ${ }^{131}$ parece indicar que si bien Augusto había entregado a Juba II el gobierno de Mauretania en el año 25 a.C., el año de la muerte de Boco II, el año 33 a.C. era una fecha importante dentro la historia de la monarquía Mauritania. ${ }^{132}$

Octaviano proyectó, sin duda, en un primer momento, anexionar definitivamente Mauretania al estado romano y transformarlo en parcela del ager publicus. ${ }^{133}$ Ciertamente, Octaviano era hostil a la política que Marco Antonio había seguido en Oriente, en donde había multiplicado coronas y reinos, por lo que en Mauretania hizo lo contrario a su competidor, y decidió no dar un sucesor a Boco II y anexionar el reino. ${ }^{134}$ Asimismo, Octaviano estaba preocupado de que la Mauretania contribuyera, al igual que el África Proconsular, a la fundación de colonias con el fin de disponer el tema de los veteranos y prolongar la romanización en dirección Oeste. ${ }^{135}$ El paso a la administración directa parece revelar la voluntad de crear un conjunto norteafricano homogéneo.

El posterior cambio de la política de Augusto en relación con Oriente le hizo reconsiderar su punto de vista inicial y aprobar el principio de la existencia de Estados clientes, que eran difíciles de suprimir sin trastornar el equilibrio geopolítico de la región. ${ }^{136}$ Esto explicaría la concesión a Juba II del trono de Mauretania.

127 Roller 2003, 95.

128 Res Gestae 25, 2.

129 Dio Cass. 60, 6, 4.

130 Dio Cass. 53, 12, 4-9.

131 Alex. II 199. Mazard 156bis.

132 Madjoub 2000, 1728; Roller 2003, 95.

133 Hugoniot 2000, 49.

134 Mackie 1983, 339; Coltellony-Trannoy 1997b, 23; Hugoniot 2000, 49; Briand-Ponsart - Hugoniat $2006,49$.

135 Coltelloni-Trannoy 1997b, 24; Hugoniot 2000, 49.

136 Hugoniot 2000, 49; Briand-Ponsart - Hugoniat 2006, 49. 
Pero no existe de manera aparente ninguna organización provincial, ni que se confiase Mauretania a dos prefectos del orden ecuestre: según Gsell, Octaviano habría delegado su autoridad a estos dos prefectos, uno en Mauretania occidental y otro en Mauretania oriental, partición que habría así anticipado un siglo la división definitiva decidida por el emperador Claudio después de la anexión. ${ }^{137}$ Esta teoría se apoyaba en la lectura de ciertas monedas ${ }^{138}$, en la que se creía poder leer el nombre de un magistrado romano, pero en realidad se trata de un monarca mauretano. ${ }^{139} \mathrm{Si}$ Octaviano tuvo esta intención, su diseño corresponde bien a la situación geográfica e histórica de este país unificado desde hacía poco tiempo, pero no existe ningún documento que corrobore una organización de este tipo. ${ }^{140}$

Mauretania no sería ni un protectorado ni una provincia: no pagaría tributo a Roma como lo hacían los reinos clientes, ni fue sometida a impuestos. Por tanto, Octaviano estuvo en una prudente expectativa antes de tomar una decisión. Mauretania tendría un estatuto espacial, pero éste no puede ser determinado hoy en día, ${ }^{141}$ y estaría pues en un limbo jurídico, ${ }^{142}$ algo que no parece viable.

De esta forma, nos encontramos como al principio, y el territorio mauretano es considerado durante el periodo de los años 33-25 a.C. alternativamente como un protectorado ${ }^{143}$ o una provincia. ${ }^{144}$ De hecho, como señala Benabou, ${ }^{145}$ es difícil definir el estatuto administrativo exacto de este territorio.

Gsell no estaba de acuerdo en considerar que Mauretania fuese una provincia, ${ }^{146}$ pues las colonias que fundó Octaviano en este territorio no figuran en su informe oficial de su reinado, ${ }^{147}$ debido a que no se habrían establecido en una verdadera provincia. Mackie advierte que el término Africa podría no sólo incluir la provincia romana de este nombre, sino todo el territorio africano hasta la costa atlántica, ${ }^{148}$ aunque Coltelloni-Trannoy está en contra de esta última opinión, ya que las fuentes diferencian claramente Africa de Mauretania. ${ }^{149}$

Asimismo, Mauretania no aparece mencionada entre las provincias que obedecían a Octaviano en el año 32 a.C., ${ }^{150}$ y Dión Casio no la menciona entre las provincias que existían en el año 27 a.C. ${ }^{151}$

137 Gsell 1928b, 201; Romanelli 1959, 161; LeVeau 1982, 700; García-Gelabert 1994, 1203.

138 RPC I 873-876.

139 Amela 2012, e.p.

140 Pavis d’Escurac 1982, 224; Coltellony-Trannoy 1997b, 25; Hugoniot 2000, 49; Madjoub 2000, 1727.

141 HugONIOT 2000, 49.

142 Roller 2003, 98.

143 Hugoniot 2000, 50; Corbier - Griesheimer 2005, 47; Briand-Ponsart - Hugoniat 2006, 49.

144 MaCKIE 1983, 336; Amandry 1987, 2.

145 Benabou 1976, 55.

146 Gsell 1928b, 201.

147 Res Gestae 5, 35-36.

148 MACKIE 1982, 351.

149 Coltelloni-Trannoy 1997b, 27.

150 Res Gestae 5, 5-6: [provi]nciae Galliae, Hispaniae, Africa, Sicilia, Sardinia.

151 Dio Cass. 53, 12, 4-6. Gsell 1928b, 201; Romanelli 1959, 161; Gruen 1996, 168; Whitakker 1996, 591; Hugoniot 2000, 49; Madjoub 2000, 1728. 
Pero para diversos investigadores los anteriores no son argumentos convincentes. Augusto pudo, en vista de los posteriores acontecimientos, pasar por alto la anexión inicial de Mauretania. La lista de Dión Casio puede ser sospechosa de anticiparse dos años en el restablecimiento del gobierno monárquico en Mauretania, aunque quizás este territorio nunca fue una prouincia en el sentido estricto del término. Así, habría que considerar que Octaviano anexionó en un principio Mauretania hasta que posteriormente cambio de opinión, o incluso que este territorio se encontraba "fuera de la ley", a la vez propiedad romana pero virgen de toda implantación romana. ${ }^{152}$

Es posible que existiera un partido mauro hostil contra las ambiciones de Octaviano en Mauretania durante los años 33-30 a.C. Así, la muerte de Bogud por Agripa sería una medida tomada por Octaviano para evitar toda aspiración eventual a retomar el poder, que debió ser acompañado de una intervención militar atroz, ${ }^{153}$ y la fundación de las colonias anteriormente mencionadas en este territorio finalizaría la operación. Sería en este periodo cuando los gétulos ocuparían parte del espacio de los mauros, con el consentimiento de Octaviano. ${ }^{154}$ Es posible que si Juba II no fue instalado inmediatamente como rey de Mauretania tras la muerte de Boco II, fue debido quizás al temor a una violenta reacción de los mauros. ${ }^{155}$

Sea como fuere, Octaviano no renunció a la herencia de Boco II, pues ya hemos visto que en las Res Gestae el territorio de Mauretania estaba de su lado. ${ }^{156}$ Pero, quizás, debido a los problemas que planteaban los mauros, y lo costoso de mantener un fuerte ejército en Mauretania, Augusto dio la independencia al territorio. ${ }^{157}$

Como señalan Romanelli y Benabou, ${ }^{158}$ la anexión pura y simple de un territorio donde se sentía apenas la presencia romana sería prematura, e incluso peligrosa en la medida donde, exigiendo la extensión sobre vastos espacios de un nuevo dispositivo de defensa, ésta habría constituido un problema para la pacificación y la integración de la antigua Numidia. Así pues, Octaviano no decidió anexionarse Mauretania, debido a que esta medida podía considerarse inadecuada o prematura por la condición del país, y el período del interregno seguramente demostraría la realidad de este aserto. Para que en un futuro cayera bajo dominio romano, se fundaron colonias que se convirtieron en centros de irradiación de la romanidad y de atracción de los indígenas.

Pero, entonces, ¿qué ocurrió en Mauretania durante el período del interregno?. Desde luego, es evidente que el territorio estaba nominalmente bajo el control de Roma, como las monedas y las fundaciones coloniales parecen indicar. Por tanto, es muy posible que Mauretania, si no fue una provincia propiamente dicha, independiente, fuese integrada a otra, a África o, incluso mejor, a la Hispania Ulterior.

Más bien, nuestra opinión es que en un primer momento Mauretania fue declarada provincia, y pasó a ser dirigida por el gobernador de la Hispania Ulterior.

152 Mackie 1983, 336-337; Coltelloni-Trannoy 1997b, 26-27.

153 Madjoub 2000, 1728-1729.

154 Madjoub 2000, 1732.

155 WhitakKer 1996, 591.

156 Romanelli 1959, 162.

157 Madjoub 2000, 1731.

158 Romanelli 1959, 162 y Benabou 1976, 48-49. 
La evolución de los acontecimientos haría que Augusto replantease su decisión y entregaría entonces Mauretania a Juba II.

Esta solución no tiene por qué extrañar, pues ya tiene antecedentes en época republicana, especialmente con respecto a la provincia del Ilírico, que dependía muchas veces del gobernador de Macedonia o el de la Galia Cisalpina, como recuerda el famoso caso de César, quien fue a la vez gobernador de Galia Cisalpina, el Ilírico y la Galia Transalpina, ${ }^{159} \mathrm{o}$ el de su asesino, M. Junio Bruto ( $p r .44$ a.C.), quien a su vez tenía bajo su mando Macedonia, Aquea (Grecia) y el Ilírico. ${ }^{160}$

De hecho, en el caso de César, la única fuente contemporánea, Cicerón, señala claramente que Ilírico formaba parte de la Galia Cisalpina. ${ }^{161}$ No en vano el que César no encontrara tropas en el Ilírico es un indicativo de que ésta todavía no era una provincia independiente en el sentido administrativo de la palabra; el Ilírico, incluso después de la muerte del Dictador, sólo sería una provincia de carácter militar. ${ }^{162}$

Que Roma tenía provincias que tuvieron un carácter efímero, sin entrar en el espinoso asunto del significado (o significados) del término prouincia, que ya por sí sólo merece un estudio aparte, no sólo parece probarlo el célebre caso peninsular de la Transduriana Prouincia, conocida por el llamado "Edicto de El Bierzo" o "Bronce de Bembibre", ${ }^{163}$ sino otra provincia más desconocida, el Quersoneso Cénico, mencionado en la lex de provinciis praetoriis del año 101 a.C. (RS 12), y que tenía el mismo gobernador que la provincia de Macedonia; esta provincia parece que sólo incluía el territorio de los tracios Caeni. ${ }^{164}$ Ambas provincias, y no debe ser casualidad, se conocen gracias únicamente a la epigrafía. Por desgracia, no podemos conocer los pormenores de su historia.

Posiblemente, el reino de Bogud se había convertido en "propiedad romana", como parece desprenderse de un testimonio de Apiano en referencia a la guerra de Yugurta. ${ }^{165}$ Octaviano acordaría la administración a Boco II, como anteriormente Escipión el Africano había efectuado con Masinisa al entregarle los territorios de Sifax II. ${ }^{166} \mathrm{Al}$ tratarse de un fragmento de una obra hoy perdida, es difícil valorar su verdadero alcance, pero muestra que todo aquel territorio que Roma obtenía por la fuerza de las armas se consideraba como propio, aunque se entregase a un monarca aliado. Desde este punto de vista, tras la muerte de Boco II, es lógico que, si no había heredero legítimo, el territorio pasara a manos de Roma.

Por ello, Octaviano, luego Augusto, decidió anexionar Mauretania, para cuyo control efectivo realizó una serie de fundaciones coloniales; posiblemente, pasó a

159 App. $B C 2$, 13. Caes. $B G$ 2, 35, 2; 3, 7, 1; 5, 1, 5. Cic. prov. cos. 36-37; Sest. 135; Vat. 35-36. Dio Cas.. 38, 8, 5. Eutrop. 6, 17, 1. Oros. 6, 7, 1. Plut. Caes. 14, 10; Cato 33, 5; Crass. 14, 4; Pomp. 48, 4. Suet. DJ 22, 1. Vell. 2, 44.

160 App. BC 3, 79; 4, 58; Cic. Ad Brut. 2, 4, 4; 5, 2; Phil. 10ss.; 13, 30. Dio. Cas.. 46, 40, 3; 47, 22. Cf. Cic. Fam. 12, 4, 2.

161 Cic. prov. cos. 3 y 36. Dzino 2010, 82.

162 SASEL Kos 2005,337 y 340.

$163 A E 1999,915=A E 2000,760=A E 2001,1214=H E p 7,1997,378=H E p$ 8, 1998, 325 = HEpOL 16498.

164 Crawford 1996, 204.

165 App. Num. 4.

166 Cf. Liv. 30, 44, 12; 37, 53, 22. GhaZı-Ben Maïssa 1996, 1416. 
depender del gobernador de Hispania Ulterior (luego, Bética). Tras la victoria de Actium y la posterior anexión de Egipto y la reorganización de Oriente, Augusto cambia de opinión y devuelve la independencia al territorio bajo la batuta de un rey-cliente, Juba II.

\section{BIBLIOGRAFÍA}

AmAndRY, M.

(1984): "Notes de numismatique africaine, I", $R N$ 26, 85-94.

(1987): “Tingi (32 av. J.-C.-29 après)", [en] H. Huvelin - M. Christol - G. Gautier (eds.), Mélanges de numismatique offerts à Pierre Bastien à l'occasion de sa 75 e anniversaire, NR, Wetteren, 1-14.

(1991-1993): "Bilan de recherches récentes sur le monnayage «romain» de Maurétanie", ANum 21-23, 239-246.

(2000): "Transformation des villes indigènes en villes romaines en Maurétanie: apport de la numismatique", [en] M. P. García-Bellido - L. Callegarin (coords.), Los cartagineses y la monetización del Mediterráneo Occidental, CSIC, Madrid, 53-58.

Amela Valverde, L.

(2001): “C. Asinio Polión en Hispania", Iberia 4, 87-110.

(2009): Hispania durante el Segundo Triunvirato (44-30 a.C.), Signifer Libros, Madrid (=Aquila Legionis 11).

(2012): "Emisiones reales de Mauretania (49-25 a.C.)", Numisma 256, e.p.

Benabou, M. (1976): La résistance africaine à la romanisation, François Maspero, Paris.

Bernal, D. ET ALII (2011): "La cronosecuencia de Tamuda. Actividades arqueológicas en desarrollo del Pal estratégico (Campañas 2009 y 2010)", [en] Seminario Internacional 'Arqueología y Turismo en el Círculo del Estrecho', Museo Arqueológico de Tetuán, Madrid - Cádiz, 276-319.

BlázQuez Martínez, J. M.

(1961): "Relaciones entre Hispania y el norte de África durante el gobierno bárquida y la conquista romana, 237-19 a.d.C.", Saitabi 11, 21-43.

(1975): Ciclos y temas de la Historia de España: la Romanización, II. La Sociedad y la Economía en la Hispania romana, Istmo, Madrid.

Briand-PonsarT, C. - Hugoniot, C. (2006): L'Afrique romaine de l'Atlantique à la Tripolitaine 146 av. J.-C.-533 ap. J.-C., Armand Colin, Paris.

Burnett, A. - AmAndry, M. - Ripollès, P. P.

(1992): Roman Provincial Coinage. Volume I. From the death of Caesar to the death of Vitellius (44 B.C.-A.D. 69). Part I: Introduction and Catalogue, British Museum Press Bibliothèque Nationale de France, London-Paris (=RPC I).

(1998): Roman Provincial Coinage. Volume I. From the death of Caesar to the death of Vitellius (44 B.C.-A.D. 69). Part I: Introduction and Catalogue. Supplement, I, British Museum Press - Bibliothèque Nationale de France, London-Paris (=RPC I).

Callegarin, L. (2002): "Considérations sur le périple sertorien dans la zone du détroit de Gibraltar (81-78 av. J.-C.)”, Pallas 60, 11-43.

CAmps, G. (2001): “Grande ou Petite Numidie?”, [en] Chr. Hambdoune (ed.), Ubique Amici. Mélanges offerts à Jean-Marie Lassère, Université Paul-Valéry, Montpellier, 67-84. 
Carcopino, J. (1943): Le Maroc antique, Gallimard, Paris.

Corbier, P. - Griesheimer, M. (2005): L'Afrique romaine 146 av. J.-C.-439 ap. J.-C., Ellipses, Paris.

Coltelloni-Trannoy, M.

(1997a): "Les liens de clientèle en Afrique du Nord, du IIe siècle av. J.-C. jusqu'au début du Principat", $B C T H(B)$ 24, 59-82.

(1997b): Le royaume de Maurétanie sous Juba II et Ptolémée (25 av. J.-C. - 40 ap. J.-C), CNRS, Paris.

(1999): “Les représentations de l'Africa dans les monnayages africains et romains à l'époque romaine", [en] S. Lancel (ed.), Afrique du nord antique et médiévale. Numismatique, langues, écritures et arts du livre, spécificité des arts figurés. Actes du VIIe colloque international sur l'Histoire et l'archéologie de l'Afrique du nord, Éditions du Comité de travaux historiques et scientifiques, Paris, 67-91.

(2005): "Rome et les rois «amis et alliés du peuple romain» en Afrique (Ier siècle av. J.-C./ Ier siècle ap. J.-C.)", Pallas 68, 117-144.

Curchin, L. A. (1996): España romana. Conquista y asimilación, Gredos, Madrid.

Decret, F. - Fantar, M. (1998): L'Afrique du Nord dans l'Antiquité. Histoire et civilisation des origines au Ve siècle, Payot, Paris.

Depeyrot, G. (1999): Zilil I. Colonia lulia Constantia Zilil. Étude du numéraire, École française de Rome, Rome.

Dzino, D. (2010): Illyricum in Roman Politics 229 BC-AD 68, Cambridge University Press, Cambridge.

EuzennAt, M. (1966): "Le roi Sosus et la dynastie maurétanienne", [en] Mélanges d'archèologie, d'épigraphie et d'histoire offerts à Jérôme Carcopino, Librairie Hachette, Vendome, Paris, 333-339.

Freyburger, M.-L. - Roddaz, J.-M. (1994): Dio Cassius. Histoire Romaine, Livres 48 et 49. Texte établi, traduit et annoté par..., Les Belles Lettres, Paris.

García-Gelabert, M. P. (1994): "La colonización romana en Hispania y Africa en época de César y Augusto", [en] A. Mastino - P. Ruggieri (eds.), L'Africa romana. Atti del X Convegno di studio, II, Archivio Fotografico Sardo, Sassari, 1189-1205.

Gascou, J. (1974): "Note sur l'évolution du statut juridique de Tánger entre 38 avant J.-C. et le règne de Claude", AntAfr 8, 67-71.

Ghazzi Ben Maïssa, H. (1996): "Les origines du royaume d'Ascalis", [en] M. Khanoussi - P. Ruggieri - C. Vismara (eds.), L'Africa romana. Atti del XI Convegno di studio, III, Il Torchietto, Ozieri, 1403-1416.

Gozalbes Cravioto, E.

(1988): “La piratería en el Estrecho de Gibraltar en la Antigüedad”, [en] E. Ripoll Perelló (ed.), Congreso Internacional El Estrecho de Gibraltar. Actas. Tomo I. Prehistoria e Historia de la Antigüedad, I, Universidad Nacional de Educación a Distancia, Madrid, 769-778. (1997): Economía de la Mauritania Tingitana: siglos I a. de C.-II de C., Instituto de Estudios Ceutíes, Ceuta.

(2003): "El intento de saqueo del Herakleion gaditano y la rebelión de los habitantes de Tingi”, [en] Actas del III Congreso de Historia de Andalucía. Historia Antigua, Publicaciones Obra Social y Cultural Cajasur, Córdoba, 273-282. 
(2011): "Iulia Traducta y las colonias de Augusto en el norte de Marruecos", [en] J. González - J. C. Saquete (eds.), Colonias de César y Augusto en la Andalucía, L'Erma di Bretschneider, Roma, 249-272.

Grant, M. (1969²): From Imperium to Auctoritas. A Historical Study of Aes Coinage in the Roman Empire 49 B.C.-A.D. 14, Cambridge University Press, Cambridge.

Grattarolo, P. (1990): I cesariani dalle idi di marzo alla costituzione del secondo triunvirato, Tirrenia, Torino.

Gruen, E. S. (1996): "The expansion of the empire under Augustus", [en] The Cambridge Ancient History. Second Edition. Volume X. The Augustan Empire, 43 B.C.-A.D. 69, Cambridge University Press, Cambridge, 147-197.

GSELL, ST.

(1928a): Histoire ancienne de L'Afrique du Nord. Tome VII. La République romaine et les rois indigènes, Librairie Hachette, Paris.

(1928b): Histoire Ancienne de L'Afrique du Nord. Tome VIII. Jules César et l'Afrique. Fin des royaumes indigénes, Librairie Hachette, Paris.

Hamdoune, Chr. (1994): "Note sur le statut colonial de Lixus et de Tanger", AntAfr 30, 81-87.

Hugoniot, Chr. (2000): Rome en Afrique: de la chute de Carthage aux débuts de la conquête arabe, Flammarion, Paris.

Kbiri-Alaoui, M. (2004): "Les établissements punico-maurétaniens de Kouass et Dchar JdidZilil (Asilah, Maroc) dans le circuit du détroit de Gibraltar", BAM 20, 195-213.

LAPORTE, J.-P. (2000): “La legio VIIa et la déduction des colonies augustéennes de Césarienne", [en] Y. Le Bohec (ed.), Les légions de Rome sous le Haut-Empire, II, Boccard, Lyon, 555-579.

Le Bohec, Y. (2005): Histoire de l'Afrique romaine, 146 avant J.-C.-439 après J.-C., Picard, Paris.

Leveau, PH. (1982): “Caesarea de Maurétanie”, $A N R W$ II 10.2, 683-738.

Mackie, N. K. (1983): “Augustan Colonies in Mauretanie”, Historia 32, 332-358.

Madjoub, M. (2000): “Octavius et la Maurétanie”, [en] M. Khanoussi - P. Ruggieri - C. Vismara (eds.), L'Africa romana. Atti del XIII Convegno di studio, II, Carocci, Roma, 17251737.

MAZARD, J.

(1953): "Numismatique de l'interrègne de Maurétanie (33 à 25 av. J.-C.)", RN 15, 13-22. (1955): Corpus Nummorum Numidiae Mauretaniaque, Arts et Métiers, Paris.

Pavis D’Escurac, H. (1982): “Les méthodes de l'impérialisme romain en Maurétanie de 33 avant J.-C. à 40 après J.-C.", Ktèma 7, 221-233.

Ponsich, M. (1982): “Tanger antique”, ANRW II 10.2, 787-816.

RHORFI, A.

(1998): Histoire prérromaine et romanisation de la Maurétanie Tingitane avant son anexión à l'empire romain, Atelier National de Reproduction de Thèses, Lille.

(2002): "La contribution de la numismatique à la connaisance de la date de la fondation coloniale de Tingi", [en] M. Khanoussi - P. Ruggieri - C. Vismara (eds.), L'Africa romana. Atti del XIV convengo di studio. Lo spazio marítimo del Mediterráneo occidentale: geografia storica ed economica, III, Carocci, Sassari, 2147-2161.

Richardson, J. S. (1998): Hispania y los Romanos. Historia de España II, Crítica, Barcelona. 
Roldán Hervás, J. M. (1978): "La guerra civil entre César y Pompeyo (49-31 a.C.)”, [en] Historia de España Antigua, II. Hispania romana, Cátedra, Madrid, 155-173.

Roller, D. W. (2003): The World of Juba II and Kleopatra Selene. Royal Scholarship on Rome's African frontier, Routledge, New York-Leiden.

Romanelli, P. (1959): Storia delle province romane dell'Africa, L'Erma di Bretschneider, Roma.

Sasel Kos, M. (2005): Appian and Illyricum, Narodni muzej Slovenije, Ljubjana.

Schмiт, P. (1977-1978): "La plus ancienne carte géographique du Maroc", BAM 11, 79-90.

Schulten, A. (1940): Fontes Hispaniae Antiquae V. Las guerras de 72-19 a. de J.C., Universidad de Barcelona, Barcelona.

Whittaker, C. R. (1996): "Roman Africa: Augustus to Vespasian", [en] The Cambridge Ancient History. Second Edition. Volume X. The Augustan Empire, 43 B.C.-A.D. 69, Cambridge University Press, Cambridge, 586-618. 